\title{
Paraneoplastic Cerebellar Degeneration and Lambert-Eaton Myasthenic Syndrome Associated with Neuroendocrine Carcinoma of the Oropharynx
}

\author{
Junji Takasugi ${ }^{1}$, Munehisa Shimamura ${ }^{1}$, Toru Koda ${ }^{1}$, Toshihiro Kishikawa ${ }^{2}$, \\ Atsushi Hanamoto ${ }^{2}$, Hidenori Inohara ${ }^{2}$, Kazuaki Sato ${ }^{3}$, Eiichi Morii ${ }^{3}$, Masakatsu Motomura ${ }^{4}$, \\ Manabu Sakaguchi ${ }^{1}$, Yuji Nakatsuji ${ }^{1}$ and Hideki Mochizuki ${ }^{1}$
}

\begin{abstract}
:
Paraneoplastic cerebellar degeneration and Lambert-Eaton myasthenic syndrome (PCD-LEMS) are usually associated with small-cell lung carcinoma (SCLC). PCD-LEMS with extrapulmonary non-SCLC tumors; however, has not been previously reported. A 78-year-old man presented with dysarthria, dysphagia, staggering gait, and lower extremity muscle fatigue. He was diagnosed with PCD-LEMS associated with neuroendocrine carcinoma of the oropharynx, based on the histological findings of the biopsy, the existence of antibodies against P/Q-type voltage-gated calcium channels, and an incremental response of the compound muscle action potentials during repetitive nerve stimulation tests. Thus, PCD-LEMS should be included in the differential diagnosis of neurological dysfunction, even in extrapulmonary non-SCLC patients.
\end{abstract}

Key words: Lambert-Eaton myasthenic syndrome (LEMS), paraneoplastic cerebellar degeneration (PCD), antibodies against P/Q-type voltage-gated calcium channels (VGCC), neuroendocrine carcinoma, oropharyngeal cancer

(Intern Med 57: 587-590, 2018)

(DOI: 10.2169/internalmedicine.9333-17)

\section{Introduction}

Lambert-Eaton myasthenic syndrome (LEMS) is a rare autoimmune disorder of neuromuscular junction transmission, which often presents with clinical features of proximal muscle weakness, diminished deep tendon reflexes, and autonomic dysfunction. Since more than half of patients have them, LEMS is often associated with tumors $(1,2)$, especially small-cell lung carcinoma (SCLC). Antibodies to $\mathrm{P} /$ Q-type voltage-gated calcium channels (VGCC), which have been detected in $85-95 \%$ of patients with LEMS, play a significant role in its pathogenesis (3-5). The antibodies inhibit the release of acetylcholine at the neuromuscular junction, which results in muscle weakness.
These antibodies are also associated with paraneoplastic cerebellar degeneration (PCD), regardless of whether PCDrelated autoantibodies, such as anti-Yo, $\mathrm{Hu}$, or Ri antibodies, are present in the serum (6). Although the precise mechanism by which PCD is caused in LEMS remains controversial, Fukuda et al. (6) reported that antibodies directed against P/Q-type VGCCs reduced the number of these channels in the molecular layer of the cerebellum. Importantly, a cohort of Japanese patients with PCD and LEMS (PCDLEMS) reportedly had SCLC and high titers of P/Q-type VGCC antibodies (4). However, PCD-LEMS has not been reported in patients with extrapulmonary non-SCLC tumors.

We herein report a unique case of PCD-LEMS associated with a neuroendocrine carcinoma (NEC) of the oropharynx, in a patient who showed no recurrence of NEC in 16

\footnotetext{
${ }^{1}$ Department of Neurology, Osaka University Graduate School of Medicine, Japan, ${ }^{2}$ Department of Otorhinolaryngology-Head and Neck Surgery, Osaka University Graduate School of Medicine, Japan, ${ }^{3}$ Department of Pathology, Osaka University Graduate School of Medicine, Japan and ${ }^{4}$ Department of Electrical and Electronics Engineering, Faculty of Engineering, Nagasaki Institute of Applied Science, Japan Received: April 9, 2017; Accepted: June 4, 2017; Advance Publication by J-STAGE: November 1, 2017 Correspondence to Dr. Hideki Mochizuki, hmochizuki@neurol.med.osaka-u.ac.jp
} 


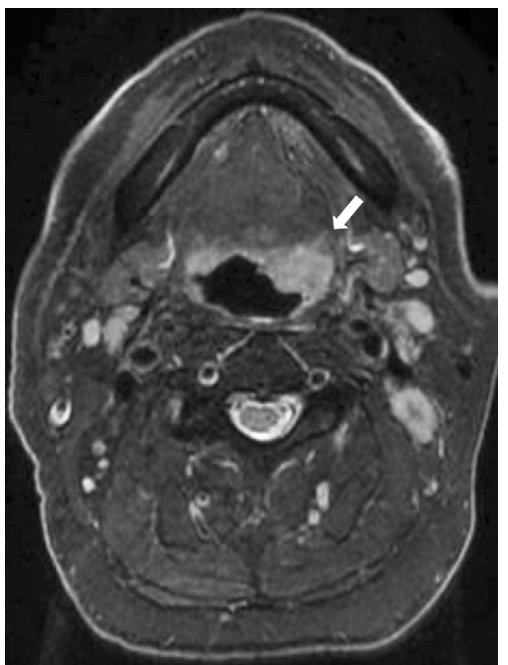

Figure 1. Magnetic resonance T2-weighted "iterative decomposition of water and fat with echo asymmetry and least squares estimation" (IDEAL) water imaging showed a 3.8 $\times 1.8$ $\mathrm{cm}$ tumor on the left side of the base of the tongue (arrow).

months of follow-up.

\section{Case Report}

The patient was a 78-year-old man with a 60-pack-peryear smoking history and no history of alcohol consumption, whose gait gradually became unsteady. In the following months, he also suffered from dysarthria, dysphagia, and lower extremity muscle fatigue during walking. At five months after the onset of symptoms, he consulted an otolaryngologist for a tumor on the left side of the base of tongue and swelling of the cervical lymph nodes. Magnetic resonance T2-weighted "iterative decomposition of water and fat with echo asymmetry and least squares estimation" (IDEAL) water imaging revealed a $3.8 \times 1.8 \mathrm{~cm}$ tumor of the left side of the base of the tongue (Fig. 1). Positron emission tomography-computed tomography (PET-CT) showed multiple swollen lymph nodes in the neck and mediastinum. There were no other distant metastases. The histological examination of an oropharynx biopsy specimen showed that the cells were arranged in clusters or sheets, which mostly consisted of atypical cells, with a high nuclear-cytoplasmic ratio (Fig. 2A), which were consistent with the features of small-cell type cancer. Immunohistochemically, the tumor cells were positive for cytokeratin AE1/AE3, synaptophysin (Fig. 2B), p16 and neural cell adhesion molecule (CD56, Fig. 2C), and negative for cytokeratin 5/6, p63, p40, and chromogranin A. The Ki-67 labeling index was approximately $70 \%$ (Fig. 2D). Thus, the patient was diagnosed with small-cell neuroendocrine carcinoma (G3) of the oropharynx (cT2N2bM0, clinical stage IVA). The levels of the serum tumor markers were within the normal limits.

He was admitted to our hospital to undergo chemoradiotherapy seven months after the onset of symptoms. During hospitalization, his staggering gait and lower extremity mus- cle fatigue remained. A neurological examination revealed proximal muscle weakness of all limbs (grade 4 on the Medical Research Council scale) and the absence of deep tendon reflexes. He exhibited saccadic eye movements, nystagmus, hypernasal speech, hypermetria of all limbs, staggering gait, and truncal ataxia. When assessed with the scale for the assessment and rating of ataxia (SARA) (7), his score was 13.5. He also had dry mouth, constipation, and orthostatic hypotension. His cranial nerve, sensory examinations, and cognitive functions were normal, except for dysarthria due to oropharynx carcinoma.

Nerve conduction studies demonstrated generalized low amplitude compound muscle action potentials, which increased by more than $200 \%$ after 10 seconds of maximal voluntary exercise. High frequency $(30 \mathrm{~Hz})$ repetitive nerve stimulation of ulnar nerve showed an increment of over $200 \%$ of the compound muscle action potential (CMAP) amplitude. The serum titer of anti-P/Q-type VGCC antibodies was elevated $(44.0 \mathrm{pmol} / \mathrm{L})$. The serum was negative for anti-acetylcholine receptor, anti-Yo, $\mathrm{Hu}$, and $\mathrm{Ri}$ antibodies. Magnetic resonance imaging of the head revealed no cerebellar abnormality. Based on these findings, he was diagnosed as having PCD-LEMS. He underwent combination chemotherapy with cisplatin $\left(60 \mathrm{mg} / \mathrm{m}^{2}\right)$ and etoposide $(75$ $\mathrm{mg} / \mathrm{m}^{2}$ ), and intensity-modulated radiation therapy to the left tonsil, neck, and mediastinum (70 Gy in 35 fractions). Initially one cycle of chemotherapy was performed, due to adverse events, which included neutropenia and pneumonia, this was followed by another cycle 6 months later. Pyridostigmine $(120 \mathrm{mg}$ ) was administered orally to relieve the patient's neurological symptoms. Since 3,4-diaminopyridine is not covered by health insurance in Japan, it was not administered to the patient. The patient's proximal muscle weakness gradually improved and returned to almost normal at the end of chemoradiotherapy. His cerebellar ataxia also improved (SARA score: 6.5). Follow-up PET-CT at 3 months revealed the disappearance of the oropharyngeal tumor and a reduction in the size of the swollen neck and mediastinal lymph nodes, without metastases. The oropharynx biopsy showed no sign of malignancy, indicating that a complete response had been achieved. He remained alive, without recurrence of NEC or a worsening of his neurological symptoms, after 16 months of follow-up.

\section{Discussion}

We herein describe a case of PCD-LEMS associated with small-cell NEC of the oropharynx. Since NECs usually arise from lungs and gastrointestinal tract, NEC originating in the oropharynx, such as in the present case, is uncommon (8). Histologically, NECs can be mainly classified into small cell type, large cell type, and mixed type (9). Among these various subtypes, small-cell NECs have been reported to have the potential to cause LEMS, because of their morphological and immunohistochemical similarity with SCLC (9). This hypothesis is supported by a few reports on LEMS with ex- 


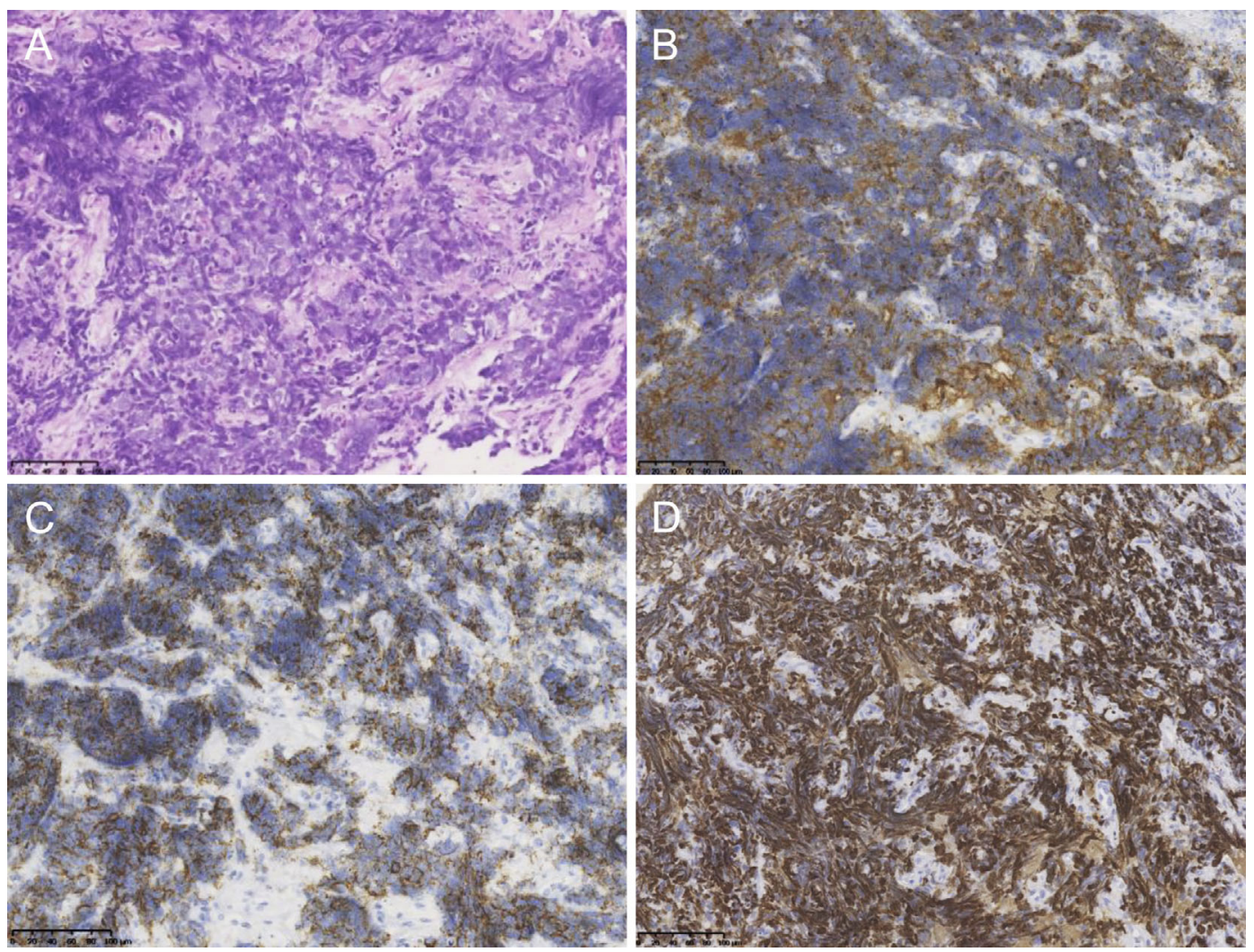

Figure 2. Histological and immunohistochemical findings of the oropharynx tumor. Hematoxylin and Eosin staining revealed atypical cells, with a high nuclear-cytoplasmic ratio, which were arranged in clusters or sheets (A). Immunohistochemical staining showed tumor cells that were positive for synaptophysin (B) and neural cell adhesion molecules (C). The Ki-67 labeling index was approximately $70 \%$ (D). The original magnification was $\times 400$. Scale bar $=100 \mu \mathrm{m}$.

trapulmonary small-cell NECs of the seminal vesicles (10) or mediastinum (11). No cases of PCD-LEMS with smallcell NECs have previously been reported.

In the treatment of LEMS associated with cancer, antitumor therapy plays a significant role in achieving remission from LEMS symptoms, such as muscle weakness (12). On the other hand, the responsiveness of PCD to anti-tumor and immunosuppressive therapy is controversial. For example, a previous report described two patients with PCD-LEMS who responded to anti-tumor therapy and plasma exchange (13), but another study revealed that three patients with PCD-LEMS who received immunotherapy showed no apparent improvement of their cerebellar symptoms (14). In the present case, chemoradiotherapy and pyridostigmine improved the patient's cerebellar ataxia, as well as his proximal muscle weakness, which is in line with the former report. One possibility is that the improvement of the patient's cerebellar symptoms might have been dependent on the degree of downregulation of the P/Q-type VGCCs in the cerebellar molecular layer. If anti-tumor therapy is administered early, the degree of downregulation might not be as severe and might recover.

Extrapulmonary NECs are known to have a poor prognosis, despite combination therapy. The median survival ranges from 4-16 months (9). In particular, small-cell NECs of the head and neck are highly aggressive and tend to develop early regional or distant metastasis (8). Interestingly, our patient with oropharynx NEC was alive with no sign of recurrence after 16 months of follow-up. This long survival without a recurrence of NEC is similar to that described in a previous study on SCLC-LEMS, which was associated with anti-P/Q-type VGCC antibodies (15). The authors suggested that the autoimmune response with anti-P/Q-type VGCC antibodies might play a role in retarding tumor growth (15). Thus, the present case highlighted that the diagnosis of LEMS would be helpful for predicting the prognosis of patients with small-cell NEC, as well as those with SCLC.

In conclusion, PCD-LEMS could be associated with extrapulmonary small-cell NECs; and cerebellar ataxia in this condition might respond to anti-tumor therapy. PCD-LEMS should be considered, even in cases with extrapulmonary small-cell NECs, in the differential diagnosis of muscular weakness and cerebellar ataxia.

The authors state that they have no Conflict of Interest (COI).

\section{References}

1. O'Neill JH, Murray NM, Newsom-Davis J. The Lambert-Eaton myasthenic syndrome. A review of 50 cases. Brain 111: 577-596, 
1988.

2. Titulaer MJ, Maddison P, Sont JK, et al. Clinical Dutch-English Lambert-Eaton myasthenic syndrome (LEMS) tumor association prediction score accurately predicts small-cell lung cancer in the LEMS. J Clin Oncol 29: 902-908, 2011.

3. Lennon VA, Kryzer TJ, Griesmann GE, et al. Calcium-channel antibodies in the Lambert-Eaton syndrome and other paraneoplastic syndromes. N Engl J Med 332: 1467-1474, 1995.

4. Nakao YK, Motomura M, Fukudome T, et al. Seronegative Lambert-Eaton myasthenic syndrome: study of 110 Japanese patients. Neurology 59: 1773-1775, 2002.

5. Motomura M, Lang B, Johnston I, Palace J, Vincent A, Newsom-Davis J. Incidence of serum anti-P/O-type and anti-Ntype calcium channel autoantibodies in the Lambert-Eaton myasthenic syndrome. J Neurol Sci 147: 35-42, 1997.

6. Fukuda T, Motomura M, Nakao Y, et al. Reduction of P/Q-type calcium channels in the postmortem cerebellum of paraneoplastic cerebellar degeneration with Lambert-Eaton myasthenic syndrome. Ann Neurol 53: 21-28, 2003.

7. Schmitz-Hübsch T, du Montcel ST, Baliko L, et al. Scale for the assessment and rating of ataxia: development of a new clinical scale. Neurology 66: 1717-1720, 2006.

8. Wang HY, Zou J, Zhou GY, Yan JQ, Liu SX. Primary small cell neuroendocrine carcinoma of the tonsil: a case report and review of the literature. Int J Clin Exp Pathol 7: 2678-2682, 2014.

9. Smith J, Reidy-Lagunes D. The management of extrapulmonary poorly differentiated (high-grade) neuroendocrine carcinomas. Semin Oncol 40: 100-108, 2013.

10. Kreiner B, Denzinger S, Ganzer R, et al. Neuroendocrine carcinoma of the seminal vesicles presenting with Lambert Eaton syndrome: a case report. J Med Case Rep 4: 320, 2010.

11. Zhang K, Liu W, Li Y, Zhang K, Gao X, Wang J. Mediastinal small cell cancer associated with Lambert-Eaton myasthenic syndrome: a case report. Exp Ther Med 10: 117-120, 2015.

12. Titulaer MJ, Lang B, Verschuuren JJ. Lambert-Eaton myasthenic syndrome: from clinical characteristics to therapeutic strategies. Lancet Neurol 10: 1098-1107, 2011.

13. Mason WP, Graus F, Lang B, et al. Small-cell lung cancer, paraneoplastic cerebellar degeneration and the Lambert-Eaton myasthenic syndrome. Brain 120: 1279-1300, 1997.

14. Graus F, Lang B, Pozo-Rosich $P$, Saiz A, Casamitjana R, Vincent A. P/Q type calcium-channel antibodies in paraneoplastic cerebellar degeneration with lung cancer. Neurology 59: 764-766, 2002.

15. Maddison P, Newsom-Davis J, Mills KR, Souhami RL. Favourable prognosis in Lambert-Eaton myasthenic syndrome and small-cell lung carcinoma. Lancet 353: 117-118, 1999.

The Internal Medicine is an Open Access article distributed under the Creative Commons Attribution-NonCommercial-NoDerivatives 4.0 International License. To view the details of this license, please visit (https://creativecommons.org/licenses/ by-nc-nd/4.0/).

(C) 2018 The Japanese Society of Internal Medicine Intern Med 57: 587-590, 2018 